CERN-EP/99-109

August 5, 1999

\title{
Do the Kamiokande results need neutrino oscillations?
}

\author{
P. Baillon ${ }^{1)}$
}

\begin{abstract}
Neutrino oscillations are a delicate and important subject. One needs to be sure that every aspect of it is well understood. The recent results of the Kamiokande experiment[1], indicate the possibility of $\tau$ - $\mu(-\mathrm{e}$ ?) neutrino oscillations. The period of oscillation observed by Kamiokande is not compatible with what one may deduce from the solar neutrino experiments[2]. In this letter, we examine if another mechanism could fake neutrino oscillations and could be measurement dependent.
\end{abstract}

(Submitted to Physics Letters B)

\footnotetext{
1) CERN CH-1211 Geneva 23;email:Paul.baillon@cern.ch
} 


\section{General remarks.}

The Kamiokande experiment[1] demonstrates that neutrinos coming from the cosmic ray air showers drastically change their composition when crossing the earth although they are not supposed to interact significantly with matter. From the experimental point of view, neutrino oscillations are not the only mechanism which could explain the Kamiokande results assuming that the production of neutrinos by the cosmic rays in the atmosphere of the earth is well understood. We want to point out that interference mechanisms associated with neutrino interactions may simulate neutrino oscillations and mix fully neutrino species.

\section{Coherent interaction of neutrinos with matter in the forward direction.}

If a neutrino is travelling inside the matter of earth, exchanging a $W$ with an electron or positron in the t or s channel, it can generate, by standard interaction, a neutrino of an other type plus a charged muon. The electrons are normally present in matter while pairs of electron positron can be taken from a virtual conversion of a virtual photon coming from the electrical field of the nucleon. We have the two reactions with a virtual $W$ in the s channel:

$$
\begin{aligned}
& \bar{\nu}_{\mathrm{e}} \mathrm{e}^{-} \rightarrow \bar{\nu}_{\mu} \mu^{-} \\
& \nu_{\mathrm{e}} \mathrm{e}^{+} \rightarrow \nu_{\mu} \mu^{+}
\end{aligned}
$$

and the two reactions with a virtual $W$ in the $t$ channel:

$$
\begin{aligned}
& \nu_{\mu} \mathrm{e}^{-} \rightarrow \mu^{-} \nu_{\mathrm{e}} \\
& \bar{\nu}_{\mu} \mathrm{e}^{+} \rightarrow \mu^{+} \bar{\nu}_{\mathrm{e}}
\end{aligned}
$$

The reactions on real electrons are operating above $10 \mathrm{GeV}$ for the energy of the incident neutrino while on virtual charged lepton $0.1 \mathrm{GeV}$ is enough. If we assume that the neutrino does not have any mass whichever is its species, its group velocity or its speed is equal to its phase velocity.

For massless particles, the wave packet can be described by a wave function

$$
\psi(\overrightarrow{\mathrm{r}}, \mathrm{t})=\int \mathrm{f}(\vec{p}) \mathrm{e}^{\mathrm{i} \phi} \mathrm{d}^{3} p, \phi=\frac{\overrightarrow{\mathrm{r}} \vec{p}-p c \mathrm{t}}{\hbar}
$$

$|\mathrm{f}(\vec{p})|^{2}$ is a gaussian type of function centered on $\vec{p}, \vec{p}$ is also the maximum of $|f(\vec{p})|^{2}$. $\vec{p}$ is the measurable value of the momentum of the wave packet while $\vec{p}$ is an integration variable which allows the wave packet to have a distribution in momentum and to be localized in space-time. The position of the center $\vec{r}$ of the wave packet with time is given by $\frac{\partial \phi}{\partial p_{\mathrm{i}}}=0$ when $\vec{p}=\overrightarrow{\mathrm{p}}$ which leads to

$$
\overrightarrow{\mathrm{r}}=\overrightarrow{\mathrm{p}} \mathrm{ct} / \mathrm{p}=\overrightarrow{\mathrm{v} t} \text {. }
$$

The phase variation of the plane wave associated to the maximum value of the wave packet is given by

$$
\phi(t)=\frac{\overrightarrow{\mathrm{r}} \overrightarrow{\mathrm{p}}-\mathrm{pct}}{\hbar}
$$

so using (1)

$$
\phi=\frac{\overrightarrow{\mathrm{p} p} \mathrm{ct} / \mathrm{p}-\mathrm{pct}}{\hbar}=0
$$


and does not depend upon time.

In other words with massless particles, a wave packet following different paths is still in phase with itself. It will interfere if the two paths meet at the same time. A single neutrino coming from the decay of a muon or a pion may interact with two different electrons and be looked at by the same detector. If the two path-lengths going from the decay point by the two different electrons to the detection point are the same within the final wave length, the two associated wave packets will meet accurately enough at the same time and will interfere. They do not need to be of the same kind all the time and at the same energy as soon as the energy difference does not introduce significant phase variation within the wave packet at the detection stage. An energy spread round $30 \%$ should not matter. This is quite common in optics and makes the optical interference easy to realize even with not fully monochromatic light.

What is nevertheless uncommon is, by an eventual detection of the intermediate lepton, the possibility of identifying the path chosen without destroying the phase of the outgoing neutrino of the other type. It is also different from the MSW mechanism: The outgoing wave has a different energy from the incoming wave. This mechanism cannot be assimilated to a matrix of indices of refraction [3].

\subsection{Estimation of the interference enhancement.}

Let us calculate, for a neutrino emitted in the atmosphere, the number of ordinary electrons which could contribute to a coherent exchange of neutrinos before reaching a point on its direction on the opposite side of the earth. In order to be in phase, the distance covered by the wave should be very close to the distance between the emission and the detection. They have to be within a wavelength $\lambda$. For a $20 \mathrm{GeV}$ neutrino

$$
\lambda=0.6210^{-16} \mathrm{~m}
$$

so the interaction point has to be inside an ellipsoid of large axis equal to the earth's diameter, $2 \mathrm{a}\left(2 \mathrm{a}=1.310^{7} \mathrm{~m}\right)$ and of small axis $2 \mathrm{~b}$, where

$$
\mathrm{b}=\sqrt{(\mathrm{a}+\lambda / 2)^{2}-\mathrm{a}^{2}} \approx \sqrt{\mathrm{a} \lambda}=210^{-5} \mathrm{~m}
$$

so the relevant volume is $\mathrm{V}=\frac{4}{3} \pi \mathrm{ab}^{2}$. The number $\mathrm{N}_{\mathrm{e}}$ of electrons in that volume $\mathrm{V}$ is

$$
\mathrm{N}_{\mathrm{e}}=\frac{4}{3} \pi \mathrm{ab}^{2} \times \rho_{\text {iron }} \mathrm{N}_{\mathrm{A}} / 2=\frac{4}{3} \pi \times 6.510^{6} \times\left(210^{-5}\right)^{2} \times 7.8610^{6} \times 6.02210^{23} / 2=0.2510^{29}
$$

The relevant angle of scattering $\theta$ is also given by the same condition, that is to say the total length should not differ from the distance to the source by more than a wave length:

$$
\sqrt{(\theta * a)^{2}+a^{2}}-a<\lambda \Rightarrow \theta<\sqrt{\frac{2 \lambda}{a}}
$$

The cross section for such a process is in first approximation its overall cross section $\sigma$ multiplied by the solid angle generated by $\theta<\sqrt{\frac{2 \lambda}{a}}$. The number of events with interference is the number given by the cross section without interference multiplied by the number of targets which are in phase with the process:

For one $\nu$ entering one has a probability of interaction $\mathrm{P}_{\mathbf{i}}$ within $\theta$ :

$$
\mathrm{P}_{\mathrm{i}}=\mathrm{N}_{\mathrm{e}} \times 2 \mathrm{a} \rho_{\text {iron }} \mathrm{N}_{\mathrm{A}} \sigma \frac{\lambda}{\mathrm{a}}=\frac{4 \pi}{3} \sigma\left(\rho_{\text {iron }} \mathrm{N}_{\mathrm{A}} \mathrm{a} \lambda\right)^{2}
$$


As said previously, if neutrinos have no mass, their group velocity is equal to their phase velocity. The phase of the wave is always the same as soon as we are in the middle of the wave packet. If the energies are the same within $30 \%$, the interference shall still be efficient because within the central part of the wave packet neutrinos are still in phase. We get a first estimation of the probability of oscillation $P_{o}$ for a muon neutrino at $20 \mathrm{GeV} / \mathrm{c}$ in earth through the exchange of a virtual $W$ in the $t$ channel.

The cross section for the reaction

$$
\nu_{\mu} \mathrm{e}^{-} \rightarrow \mu^{-} \nu_{\mathrm{e}}
$$

is around $\sigma=1.610^{-44} \mathrm{~m}^{2}=\left(2 \mathrm{~m}_{\mathrm{e}} \mathrm{E}_{\nu}-\mathrm{m}_{\mu}^{2}\right) \mathrm{G}_{\mathrm{f}}^{2} / \pi[4], \lambda=0.6210^{-16} \mathrm{~m}$. We have:

$$
\mathrm{P}_{\mathrm{o}}=\mathrm{P}_{\mathrm{i}}=0.24
$$

Those reactions should also occur on virtual $e^{+}$or $e^{-}$coming from a virtual $\gamma$ in the electrical field of an iron nucleus with a much higher probability and at lower energy: The electromagnetic showers associated with $\mathrm{GeV}$ electrons and photons are generated mainly by the electromagnetic interactions with the electrical field of the nucleons and not by the interactions with the electrons present in the medium. We shall have to integrate over Fermi momentum of the various constituents of the iron nucleus. At the energy of the cosmic neutrino, this will average on a large band of the angular distribution at the weak interaction vertices of the associated Feynman diagrams eliminating zero's due to angular momentum conservation. If this mechanism works, we should expect an equal number of $\nu_{\mathrm{e}} \bar{\nu}_{\mathrm{e}} \nu_{\mu} \bar{\nu}_{\mu}$ after neutrino crossing the earth, whatever the incoming neutrino type. This is close to what is observed.

Since we have multiplication factors which may be big, diagrams involving virtual $\tau$ may become effective and we may think at some production of $\nu_{\tau}$ although one is most of the time below the threshold of $\tau$ production. If this proved to exist, it could lower the up going number of $\nu_{\mathrm{e}}$ and $\nu_{\mu}$ coming from the atmosphere and crossing the earth.

In the same spirit, a reaction like:

$$
\nu_{\mathrm{e}} \mathrm{q} \rightarrow \nu_{\mathrm{e}} \bar{\nu}_{\mu} \nu_{\mu} \mathrm{q}
$$

where q represents a quark or a lepton, may explain by interference mechanism the solar neutrino flux.

\section{References}

[1] Evidence for oscillation of atmospheric neutrinos, The Super-Kamiokande Collaboration, Phys. Rev. Lett. 81 (1998) 1562-1567

[2] J. Conrad Review talk on neutrino oscillations at ICHEP-98 23-29 July 1998, proceeding of ICHEP-98 University of British Columbia Vancouver, BC. Canada

[3] L. Wolfenstein, Phys. Rev. D17 2369 (1978); S.P. Mikheyev, A. Yu. Smirnov Nu. Cim. C9 17 (1986)

[4] See for instance E.D. Commins, P.H. Bucksdaum, Weak Interactions of lepton and quarks, pages 124-126 and 310, Cambridge University Press Cambridge London U.K. 1983 ,

Review of particle physics Eur. Phys. J. C 31998 page 92 